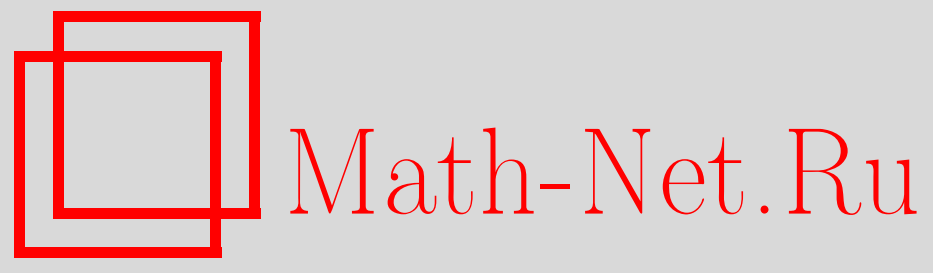

В. Л. Леонтьев, Ортогональная система Франклина и ортогональная система финитных функций в численных методах решения краевых задач, Вестн. Сам. гос. техн. ун-та. Сер. Физ.-мат. науки, 2015, номер 2, 398-404

DOI: https://doi.org/10.14498/vsgtu1414

Использование Общероссийского математического портала MathNet.Ru подразумевает, что вы прочитали и согласны с пользовательским соглашением

http: //www . mathnet.ru/rus/agreement

Параметры загрузки:

IP : 54.174 .149 .18

26 апреля 2023 г., 16:18:17

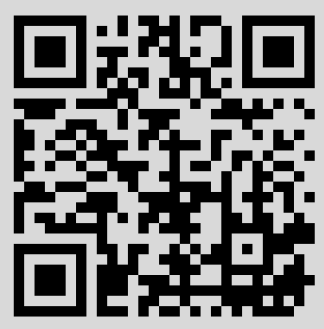


УДК 519.63, 519.651

\title{
ОРТОГОНАЛЬНАЯ СИСТЕМА ФРАНКЛИНА И ОРТОГОНАЛЬНАЯ СИСТЕМА ФИНИТНЫХ ФУНКЦИЙ В ЧИСЛЕННЫХ МЕТОДАХ РЕШЕНИЯ КРАЕВЫХ ЗАДАЧ
}

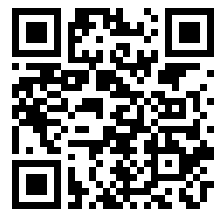

\section{В. Л. Леонтъев}

Ульяновский государственный университет, Россия, 432017, Ульяновск, ул. Л. Толстого, 42.

\begin{abstract}
Аннотация
Возможности классических рядов Фурье, связанных с тригонометрическими функциями, существенно ограничены в двумерных и трехмерных краевых задачах. Граничные условия таких краевых задач для областей с криволинейными границами часто не удается выполнить при использовании классических рядов Фурье. Решение этой проблемы дает использование ортогональных финитных базисных функций. Однако ортогональные базисные функции Хаара не являются непрерывными. Ортогональные вейвлеты Добеши имеют компактные носители, но не записываются в аналитической форме и имеют низкую гладкость. Непрерывные финитные функции Фабера-Шаудера не являются ортогональными. Ортогональные непрерывные функции Франклина не являются финитными. Здесь установлена связь ортогональной системы функций Франклина с последовательностью сеточных наборов кусочно-линейных ортогональных финитных базисных функций (ОФФ). Сформирован ряд Фурье-ОФФ на основе таких непрерывных ОФФ, который позволяет выполнять граничные условия типа Дирихле на криволинейных границах в рамках интегральных постановок краевых задач. Аналогичная проблема, связанная с удовлетворением граничных условий типа Неймана, также устраняется при дополнительном использовании смешанных интегральных постановок краевых задач. Ряд Фурье-ОФФ повышает эффективность смешанных численных методов решения краевых задач.
\end{abstract}

Ключевые слова: ортогональная система функций, ортогональные финитные функции, ряд Фурье, смешанные численные методы решения краевых задач.

doi: http://dx.doi.org/10.14498/vsgtu1414

(C) 2015 Самарский государственный технический университет.

\section{Образец для цитирования}

Л е он т ь в В. Л. Ортогональная система Франклина и ортогональная система финитных функций в численных методах решения краевых задач // Вестн. Сам. гос. техн. ун-та. Сер. Физ.-мат. науки, 2015. Т. 19, № 2. С. 398-404. doi: 10.14498/vsgtu1414.

Сведения об авторе

Виктор Леонтьевич Леонтьев (д.ф.-м.н., проф.; LeontievVL@ulsu.ru), профессор, каф. информационная безопасность и теория управления.

*Настоящая статья представляет собой расширенный вариант доклада [1], сделанного автором на Четвёртой международной конференции «Математическая физика и её приложения» (Россия, Самара, 25 августа - 1 сентября 2014). 
Применение классических рядов Фурье, связанных с тригонометрическими функциями, существенно ограничено в двумерных и трехмерных краевых задачах. Граничные условия Дирихле и Неймана часто не удается выполнить для областей с криволинейными границами при использовании тригонометрических рядов Фурье. Решение этой проблемы дает использование ортогональных базисных функций с компактными носителями. Однако ортогональные базисные функции Хаара [2] не являются непрерывными. Ортогональные вейвлеты Добеши [3] имеют компактные носители, но не записываются в аналитической форме и имеют низкую гладкость. Непрерывные финитные функции Фабера-Шаудера $[4,5]$ не являются ортогональными. Ортогональные непрерывные функции Франклина [6] не являются финитными. Исследования в области ортогональных рядов активно проводились ранее и проводятся в настоящее время [7-13]. Большое внимание при этом обращается на ортогональные ряды, связанные с финитными функциями.

Здесь установлена связь ортонормированной системы функций Франклина с последовательностью сеточных наборов ортонормированных финитных функций [14], показывающая возможность формирования рядов Фурье на основе ортонормированных финитных функций [14], предназначенных для их эффективного использования в алгоритмах численных методов решения краевых задач математической физики. Сформирован ряд Фурье-ОФФ на основе таких непрерывных ОФФ, который позволяет выполнять граничные условия типа Дирихле на криволинейных границах в рамках интегральных постановок краевых задач. Аналогичная проблема, связанная с удовлетворением граничных условий типа Неймана, также устраняется при дополнительном использовании смешанных интегральных постановок краевых задач. Выявленная связь функций Франклина и последовательности сеточных наборов ОФФ обосновывает универсальность процедуры ортогонализации [14] систем сеточных базисных функций, предложенной в 1993 году в работах В. Л. Леонтьева. Эта процедура, в отличие от классических процедур ортогонализации Грама-Шмидта и продолжения функций на более длинный интервал, сохраняет свойство финитности базисных функций. Ортонормированная система функций Франклина (нефинитных функций) [6] - исторически первый пример ортонормированного базиса пространства непрерывных функций. Система была получена в результате ортогонализации базисных функций Фабера-Шаудера $[4,5]$ с помощью процедуры Грама-Шмидта. Система функций Фабера-Шаудера представляет собой полную систему линейно независимых функций-шапочек, выборочно взятых из последовательности наборов сеточных финитных непрерывных кусочно-линейных функций-шапочек, причем каждый очередной набор таких функций порождается процедурой половинного деления предыдущей сетки на отрезке $[0,1]$. Ортогонализация, также с помощью процедуры Грама-Шмидта, функций-шапочек типа функций Фабера-Шаудера, взятых только из одного полного сеточного набора $N$ функций [14] на отдельной сетке, приводит в случае $N=5$ к нефинитным ортонормированным функциям

$$
F_{1}(x)=\left\{\begin{array}{cl}
2 \sqrt{3}(1-4 x), & x \in[0,1 / 4) ; \\
0, & x \in[1 / 4,1]
\end{array}\right.
$$




$$
\begin{aligned}
& F_{2}(x)=\left\{\begin{array}{cl}
2 \sqrt{3 / 7}(12 x-1), \quad x \in[0,1 / 4) ; \\
8 \sqrt{3 / 7}(1-2 x), \quad x \in[1 / 4,1 / 2) ; \\
0, \quad x \in[1 / 2,1] ;
\end{array}\right. \\
& F_{3}(x)=\left\{\begin{array}{cl}
-2 \sqrt{3 / 91}(12 x-1), & x \in[0,1 / 4) ; \\
2 \sqrt{3 / 91}(36 x-11), & x \in[1 / 4,1 / 2) ; \\
14 \sqrt{3 / 91}(3-4 x), & x \in[1 / 2,3 / 4) ; \\
0, & x \in[3 / 4,1] ;
\end{array}\right. \\
& F_{4}(x)=\left\{\begin{array}{cl}
2 \sqrt{3 / 1261}(12 x-1), & x \in[0,1 / 4) ; \\
-2 \sqrt{3 / 1261}(36 x-11), & x \in[1 / 4,1 / 2) ; \\
2 \sqrt{3 / 1261}(132 x-73), & x \in[1 / 2,3 / 4) ; \\
4 \sqrt{39 / 97}(4-4 x), & x \in[3 / 4,1] ; \\
-(12 x-1) / \sqrt{679}, & x \in[0,1 / 4) ; \\
(36 x-11) / \sqrt{679}, & x \in[1 / 4,1 / 2) ; \\
-(132 x-73) / \sqrt{679}, & x \in[1 / 2,3 / 4) ; \\
(492 x-395) / \sqrt{679}, & x \in[3 / 4,1] .
\end{array}\right.
\end{aligned}
$$

Каждая из этих пяти нефинитных функций является линейной комбинацией первых пяти функций Франклина и так же, как и соответствующие им первые пять функций Франклина, является частью полной системы базисных функций. Таким образом, существует возможность формирования ортонормированных базисных функций не на базе системы Фабера-Шаудера, а на базе последовательности полных сеточных наборов линейно независимых финитных функций-шапочек, позволяющей проводить ортогонализацию не только на основе процедуры Грама-Шмидта. Иной метод ортогонализации таких сеточных наборов, сохраняющий свойство финитности, имеющееся у исходных функций-шапочек, представлен в [14] и связан с изменением каждой финитной функции-шапочки без нарушения свойств непрерывности и финитности функций. При сгущении сеток их бесконечной последовательности порождаются наборы ортогональных финитных функций, число которых растет при переходе от менее густой сетки к более густой. При этом каждый такой набор представляет собой базис в соответствующем $N$-мерном подпространстве, где базисом является набор $N$ функций Франклина, число которых совпадает с числом указанных ортогональных финитных сеточных функций. Таким образом, установлена универсальность процедуры ортогонализации систем функций, предложенной в [14] и в статьях, опубликованных ранее. Ссылки на эти статьи даются в [14]. Таким образом, две классические процедуры ортогонализации систем функций: процедура Грама-Шмидта и продолжения на более длинный интервал, на основе данного исследования дополняются процедурой ортогонализации [14], отличающейся от названных классических процедур тем, что процедура ортогонализации [14] сохраняет свойство финитности функций, имеющее исключительно большое значение для вычислительной математики. Формирование обобщенного ряда Фурье на основе системы сеточных функций Франклина осуществляется обычным образом, поскольку в этой системе присутствуют функции, порождаемые всеми сетками на $[0,1]$. Напротив, ряд Фурье, связанный с ортогональными финитными функциями, на основе изложенных результатов строится как предел последовательности частичных сумм, образованных последовательностями 
наборов ортонормированных систем финитных функций, полных в соответствующих конечномерных подпространствах и построенных на последовательности сгущающихся сеток. При этом при переходе от одной частичной суммы последовательности к другой частичная сумма полностью перестраивается.

Применение названных базисных систем функций приводит к эффективным алгоритмам [15-18] смешанных численных методов исследования математических моделей - краевых или эволюционно-краевых задач. Эффективность таких алгоритмов определяется использованием смешанных вариационных постановок краевых задач или смешанных интегральных проекционных условий, поскольку в этих случаях ортогональность базисных функций на каждой отдельной сетке делает алгоритм численного метода существенно более рациональным и эффективным. Приближенное решение краевой задачи находится при проведении каждого отдельного расчета с помощью алгоритма смешанного вариационно-сеточного или смешанного проекционносеточного метода, реализованного на ЭВМ на одной конкретной сетке. Для уточнения решения сетка сгущается, на измененной более густой сетке также имеет место ортогональность сеточных финитных функций, приводящая после проведения дискретизации по пространственным переменным к системе ОДУ или к системе алгебраических уравнений в случае эллиптической задачи с более простыми структурами по сравнению с вариационно-сеточными или проекционно-сеточными методами, в которых используются неортогональные базисные функции. Так, в случае линейной эллиптической задачи после дискретизации образуется система линейных алгебраических уравнений с разреженной матрицей, которая во многих строках имеет ненулевые компоненты только на своей главной диагонали. Последнее свойство порождает рациональный алгоритм решения системы алгебраических уравнений, позволяющий сократить число вычислительных операций получения решения в несколько раз.

Применение ортогональных финитных функций приводит также к новому интегральному преобразованию [19], по эффективности не уступающему интегральному вейвлет-преобразованию, а также к рациональной математической модели сил межатомного взаимодействия [20], позволяющей снизить число арифметических операций, затрачиваемых на решение задач о движении атомов углеродных нанотрубок.

Благодарности. Работа выполнена при финансовой поддержке Министерства образования и науки Российской Федерации в рамках государственного задания.

\section{ORCID}

Виктор Леонтьевич Леонтьев: http://orcid.org/0000-0002-8669-1919

\section{БИБЛИОГРАФИЧЕСКИЙ СПИСОК}

1. Леонтьев В. Л. Ортогональная система Франклина и ортогональная система финитных функций в численных методах решения краевых задач/ Четвертая международная конферениия «Математическая физика и ее приложения»: материалы конф.; ред. чл.корр. РАН И. В. Волович; д.ф.-м.н., проф. В. П. Радченко. Самара: СамГТУ, 2014. C. 228-229.

2. Haar A. Zur Theorie der orthogonalen Funktionensysteme // Math. Ann., 1910. vol. 69, no. 3. pp. 331-371. doi: 10.1007/bf01456326. 
3. Daubechles I. Orthonormal Bases of Compactly Supported Wavelets // Commun. Pure Appl. Math., 1988. vol.41, no.7. pp. 909-996. doi:10.1002/cpa.3160410705; Daubechles I. Orthonormal Bases of Compactly Supported Wavelets / Fundamental Papers in Wavelet Theory. Princeton: Princeton University Press, 2009. pp. 564-652. doi: 10.1515/ 9781400827268.564 doi : 10.1515/9781400827268.

4. Faber G. Über die Orthogonalfunktionen des Herrn Haar// Jahresbericht der Deutschen Mathematiker-Vereinigung, 1910. vol. 19. pp. 104-112.

5. Shauder J. Eine Eigenschaft des Haarschen Orthogonalsystems // Math. Z., 1928. vol. 28, no. 1. pp. 317-320. doi : 10.1007/BF01181164.

6. Franklin P. A set of continuous orthogonal functions // Math. Ann., 1928. vol. 100, no. 1. pp. 522-529. doi: 10.1007/bf01448860 ; Franklin P. A set of continuous orthogonal functions / Fundamental Papers in Wavelet Theory. Princeton: Princeton University Press, 2009. pp. 189-196. doi: 10.1515/9781400827268.189 doi: 10.1515/9781400827268.

7. Ульянов П. Л. О рядах по системе Хаара // Докл. Акад. наук СССР, 1963. Т. 149, № 3. C. $532-534$.

8. Ульянов П. Л. О рядах по системе Хаара // Матем. сб., 1964. Т. 63(105), № 3. С. 356391.

9. Schipp F., Simon P. Investigation of Haar and Franklin series in Hardy spaces // Anal. Math., 1982. vol. 8, no. 1. pp. 47-56. doi: 10.1007/bf02073771.

10. Геворкян Г. Г. Об абсолютной и безусловной сходимости рядов по системе Франклина // Матем. заметки, 1989. Т. 45, № 3. С. 30-42.

11. Wojtaszczyk P., Woźniakowski K. Orthonormal polynomial bases in function spaces // Israel J. Math., 1991. vol. 75, no. 2/3. pp. 167-191. doi: 10.1007/bf02776023.

12. Кашин Б. С., Саакян А. А. Ортогональные рядъ. М.: АФЦ, 1999. 550 с.

13. Chen W., Cai Z., Qi D. A New Class of Orthogonal Spline Moments and Its Application // J. Inf. Comput. Sci., 2013. vol. 10, no. 14. pp. 4563-4571. doi: 10.12733/jics20102141.

14. Леонтьев В. Л. Ортогональные финитные функции и численные методы. Ульяновск: УлГУ, 2003. 178 с.

15. Леонтьев В. Л. Вариационно-сеточный метод решения задач о собственных колебаниях упругих трехмерных тел, связанный с использованием ортогональных финитных функций // Изв. РАН. МТT, 2002. №3. С. 117-126.

16. Леонтьев В. Л. Ортогональные сплайны и вариационно-сеточный метод// Матем. моделирование, 2002. Т. 14, №3. С. 117-127.

17. Леонтьев В. Л., Лукашанец Н. Ч. О сеточных базисах ортогональных финитных функций // Ж. вычисл. матем. и матем. физ., 1999. Т. 39, №7. С. 1158-1168.

18. Красильников А. Р., Леонтьев В. Л. О вариационно-сеточном методе теории пластин // Матем. моделирование, 2005. Т. 17, № 3. С. 23-34.

19. Леонтьев В. Л., Риков Е. А. Интегральные преобразования, связанные с ортогональными финитными функциями, в задачах спектрального анализа сигналов // Матем. моделирование, 2006. Т. 18, № 7. С. 93-100.

20. Леонтьев В. Л., Михайлов И. С. О построении потенциала взаимодействия атомов, основанном на ортогональных финитных функциях // Нано- и микросистемная техника, 2011. № 9. С. 48-50.

Поступила в редакцию 03/IX/2014; в окончательном варианте - 09/XI/2014; принята в печать - 11/III/2015. 
Vestn. Samar. Gos. Techn. Un-ta. Ser. Fiz.-mat. nauki

[J. Samara State Tech. Univ., Ser. Phys. \& Math. Sci.], 2015, vol. 19, no. 2, pp. 398-404

ISSN: 2310-7081 (online), 1991-8615 (print)

doi: http://dx.doi.org/10.14498/vsgtu1414

MSC: 65M60, 41A30

\title{
ORTHOGONAL FRANKLIN SYSTEM AND ORTHOGONAL SYSTEM OF FINITE FUNCTIONS IN NUMERICAL METHODS OF BOUNDARY PROBLEMS SOLVING*
}

\section{L. Leontiev}

Ulyanovsk State University,

42, L. Tolstoy st., Ulyanovsk, 432017, Russian Federation.

\begin{abstract}
Possibilities of classical trigonometric Fourier series are substantially limited in 2-D and 3-D boundary value problems. Boundary conditions of such problems for areas with curvilinear boundaries often fails when using the classical Fourier series. The solution of this problem is the use of orthogonal finite functions. However, orthogonal Haar basis functions are not continuous. The orthogonal Daubechies wavelets have compact supports, but is not written in analytical form and have low smoothness. Continuous finite Schauder-Faber functions are not orthogonal. Orthogonal Franklin continuous functions are not finite. The connection of the orthogonal Franklin functions with a sequence of grid groups of piecewise linear orthogonal finite basis functions (OFF) is established here. The Fourier-OFF series on the basis of such continuous OFF is formed. Such series allows to execute boundary conditions of Dirichlet's type on curvilinear boundaries in integral performances of boundary value problems. A similar problem is connected with a satisfaction of Neumann boundary conditions and also is eliminated in the integral mixed performances of boundary value problems. Fourier-OFF series increases the effectiveness of mixed numerical methods for boundary value problems solving.
\end{abstract}

Keywords: orthogonal system of functions, orthogonal finite functions, Fourier series, mixed numerical methods for boundary problems solving.

doi: http://dx.doi.org/10.14498/vsgtu1414

Acknowledgments. This work was supported by the Ministry of Education and Science of the Russian Federation.

ORCID

Victor L. Leontiev: http://orcid.org/0000-0002-8669-1919

(C) 2015 Samara State Technical University.

Please cite this article in press as:

Leontiev V. L. Orthogonal Franklin system and orthogonal system of finite functions in numerical methods of boundary problems solving, Vestn. Samar. Gos. Tekhn. Univ., Ser. Fiz.-Mat. Nauki [J. Samara State Tech. Univ., Ser. Phys. \& Math. Sci.], 2015, vol. 19, no. 2, pp. 398-404. doi: 10.14498/vsgtu1414. (In Russian)

Author Details:

Victor L. Leontiev (Dr. Phys. \& Math. Sci.; LeontievVL@ulsu.ru), Professor, Dept. of Information Security and Control Theory.

${ }^{*}$ This paper is an extended version of the paper [1], presented at the Mathematical Physics and Its Applications 2014 Conference. 


\section{REFERENCES}

1. Leontiev V. L. Orthogonal Franklin system and orthogonal system of finite functions in numerical methods of boundary problems solving, The 4nd International Conference "Mathematical Physics and its Applications", Book of Abstracts and Conference Materials; eds. I. V. Volovich; V. P. Radchenko. Samara, Samara State Technical Univ., 2014, pp. 228 229 (In Russian).

2. Haar A. Zur Theorie der orthogonalen Funktionensysteme, Math. Ann., 1910, vol. 69, no. 3, pp. 331-371. doi: 10.1007/bf01456326.

3. Daubechles I. Orthonormal Bases of Compactly Supported Wavelets, Commun. Pure Appl. Math., 1988, vol.41, no.7, pp. 909-996. doi:10.1002/cpa.3160410705 ; Daubechles I. Orthonormal Bases of Compactly Supported Wavelets, Fundamental Papers in Wavelet Theory. Princeton, Princeton University Press, 2009, pp. 564-652. doi: 10.1515/ 9781400827268.564 doi : 10.1515/9781400827268.

4. Faber G. Über die Orthogonalfunktionen des Herrn Haar, Jahresbericht der Deutschen Mathematiker-Vereinigung, 1910, vol. 19, pp. 104-112.

5. Shauder J. Eine Eigenschaft des Haarschen Orthogonalsystems, Math. Z., 1928, vol. 28, no. 1, pp. 317-320. doi : 10.1007/BF01181164.

6. Franklin P. A set of continuous orthogonal functions, Math. Ann., 1928, vol. 100, no. 1, pp. 522-529. doi: 10.1007/bf01448860 ; Franklin P. A set of continuous orthogonal functions, Fundamental Papers in Wavelet Theory. Princeton, Princeton University Press, 2009, pp. 189-196. doi: 10.1515/9781400827268.189 doi: 10.1515/9781400827268.

7. Ul'yanov P. L. Haar series, Sov. Math., Dokl., 1963, vol. 4, pp. 437-440.

8. Ul'janov P. L. On Haar series, Mat. Sb. (N.S.), 1964, vol.63, no. 105, pp. 356-391 (In Russian).

9. Schipp F., Simon P. Investigation of Haar and Franklin series in Hardy spaces, Anal. Math., 1982, vol. 8, no. 1, pp. 47-56. doi: 10.1007/bf02073771.

10. Gevorkyan G. G. Absolute and conditional convergence of series in Franklin systems, Math. Notes, 1989, vol.45, no. 3, pp. 200-210. doi: 10.1007/BF01158555.

11. Wojtaszczyk P., Woźniakowski K. Orthonormal polynomial bases in function spaces, Israel J. Math., 1991, vol.75, no. 2/3, pp. 167-191. doi: 10.1007/bf02776023.

12. Kashin B. S., Saakyan A. A. Ortogonal'nye riady [Orthogonal series]. Moscow, AFTs, 1999, 550 pp. (In Russian)

13. Chen W., Cai Z., Qi D. A New Class of Orthogonal Spline Moments and Its Application, J. Inf. Comput. Sci., 2013, vol. 10, no. 14, pp. 4563-4571. doi: 10.12733/jics20102141.

14. Leontiev V. L. Ortogonal'nye finitnye funktsii i chislennye metody [Orthogonal compactly supported functions and numerical methods]. Ulyanovsk, Ulyanovsk State Univ., 2003, 178 pp. (In Russian)

15. Leont'ev V. L. A variational-grid method involving orthogonal finite functions for solving problems of natural vibrations of 3D elastic solids, Mech. Solids, 2002, vol. 37, no. 3, pp. 101109.

16. Leontiev V. L. Orthogonal splines and variational-grid method, Mat. Model., 2002, vol. 14, no. 3, pp. 117-127 (In Russian).

17. Leont'ev V. L., Lukashanets N. Ch. Grid bases of orthogonal compactly supported functions, Comput. Math. Math. Phys., 1999, vol. 39, no. 7, pp. 1116-1126.

18. Krasil'nikov A. R.; Leontiev V. L. On the variation-grid method of the plate theory, Mat. Model., 2005, vol. 17, no. 3, pp. 23-34 (In Russian).

19. Leontiev V. L., Rikov E. A. Integral transforms associated with orthogonal finite functions in the spectral analysis of signals, Mat. Model., 2006, vol. 18, no. 7, pp. 93-100 (In Russian).

20. Leontiev V. L., Mikhaylov I. S. On the Building the Potential of the Atomic Interaction Based on Orthogonal Finite Functions, Nano- $i$ mikrosistemnaia tekhnika, 2011, no.9, pp. 48-50 (In Russian). 